\title{
Corneal Involvement in Herpes Zoster Maxillaris: A Rare Occurrence
}

\author{
Shovna Dash, Gayatree Mohanty \\ Department of Ophthalmology, Kalinga Institute of Medical Sciences \& Pradyumna Bal Memorial Hospital, Bhubaneswar, \\ Odisha, India.
}

\author{
Corresponding Author: \\ Dr. Gayatree Mohanty \\ Email:mgayatree@gmail.com \\ This is an Open Access article distributed \\ under the terms of the Creative Commons \\ Attribution License (creativecommons.org/ \\ licenses/by/3.0). \\ Received \\ Accepted \\ Published \\ May 3, 2020 \\ July 20, 2020 \\ August 20, 2020
}

\begin{abstract}
Background: Herpes zoster ophthalmicus occurs due to reactivation of latent varicellazoster virus in the trigeminal ganglion, commonly involving the ophthalmic division. To the best of our knowledge, ocular involvement in zoster cases affecting the maxillary division have been rarely reported. Case Report: We present two young females with herpes zoster ophthalmicus involving the maxillary division with corneal involvement. Conclusion: All cases of zoster infection should be referred to an ophthalmologist for early intervention and prevent sequelae like corneal opacity. Maxillary zoster may sometimes precede ophthalmic zoster and zoster ophthalmicus may occur without skin rashes, involving only the cornea or iris. .
\end{abstract}

Keywords: Cornea, Corneal Opacity, Herpes Zoster Ophthalmicus, Ophthalmologists, Varicella Zoster Virus Infection.

\section{Introduction}

Herpes zoster ophthalmicus is a neurocutaneous disorder caused by the Human Herpes Virus-3, the same virus that causes varicella. Approximately $13 \%$ of the patients present with infections involving any of the three branches of the trigeminal nerve. However involvement of the maxillary division is quite rare, approximately seen in $1.7 \%$ of the cases. We come across innumerable cases of herpes zoster infection in our clinic and are well-versed with it. It's easy to spot prototype cases of herpes zoster ophthalmicus but it is rare to encounter cases with only the maxillary division being involved and detect ocular involvement. Here is a brief report of two young females with similar findings.

Our hospital based observational study has been approved by the Institutional Ethics Committee according to the tenets of the declaration of Helsinki. Written informed consent was taken from the patient for collection of his/ her details and images.

\section{Case Report}

Two young females presented with complaints of decreased vision in left eye with vesicular eruptions over their left cheek, side of nose and upper lip. Both patients had multiple eruptions in various stages on the left side of face, over the malar eminence, side of nose, tip of nose, upper lip and near the angle of mouth. There was mild lower lid edema and ciliary congestion in the left eye in both the cases. A corneal sensation of the left eye was diminished. Fluorescein staining was negative in both cases. Ocular movements and intraocular pressure were normal.

Our first case had a diffuse patch of anterior stromal infiltration in the lower one-third of the cornea without epithelial involvement and best corrected visual acuity 6/6(p) and 6/18 [Fig.1]. In second case, a limbal phlyctenular lesion was 
associated with conjunctival congestion at nine o'clock position and best corrected visual acuity 6/6 and 6/12(p) [Fig.2].

A clinical diagnosis of maxillary zoster was made based on the typical, sharp limitation of vesicular eruptions within a specific area of neural distribution. There was no oral mucosal ulceration. Otorhinolaryngology and dental examination supported the diagnosis of maxillary zoster. Routine investigations were done. Blood sugar was normal. HIV and syphilis were ruled out by serological tests. Oral acyclovir $800 \mathrm{mg} 5$ times a day for 7 days was prescribed, supplemented by NSAIDs. Antibiotic drops were prescribed along with antibiotic ointment for the lesions.

\section{Discussion}

Herpes zoster ophthalmicus occurs due to reactivation of latent varicella-zoster virus (VZV) in the trigeminal ganglion. The reactivated virus has a predilection for the ophthalmic division of the trigeminal nerve [1]. The ophthalmic division of the trigeminal nerve is 20 times more commonly involved than the maxillary or mandibular nerves. This case report is regarding herpes zoster infection of the maxillary nerve, which is quite rare, approximately seen in $1.7 \%$ of the cases $[2,3]$.

The ophthalmic division of the trigeminal nerve divides into three main branches: the frontal nerve, the lacrimal nerve and the nasociliary nerve. The nasociliary branch innervates the ciliary body, iris, cornea and conjunctiva. Its terminal branch is the anterior ethmoidal nerve, which innervates the sides of the tip of the nose (alae nasae) via the external nasal nerve. Hutchinson observed that ocular involvement is more commonly seen if the herpes zoster opthalmicus rash involves the alae nasae [4]. Up to $85 \%$ of such cases may develop ocular involvement [5]. If Hutchinson's sign is absent ocular involvement is less likely, however

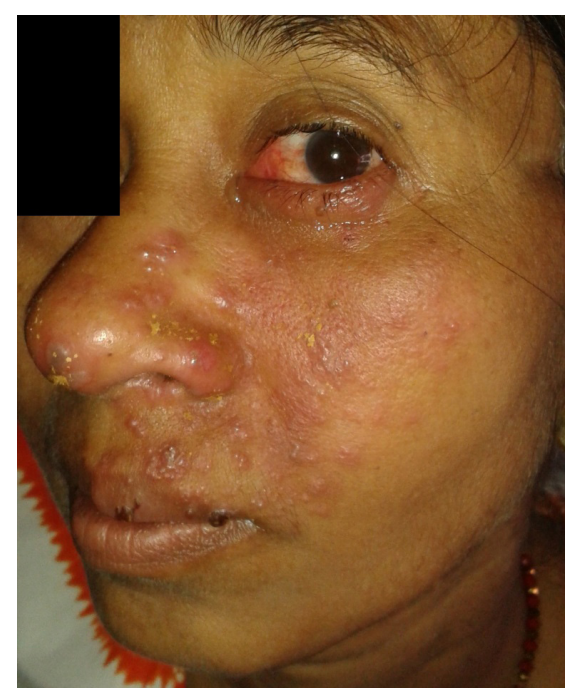

Fig.1: Maxillary involvement with Hutchison sign and lower $1 / 3^{\text {rd }}$ of corneal involvement.

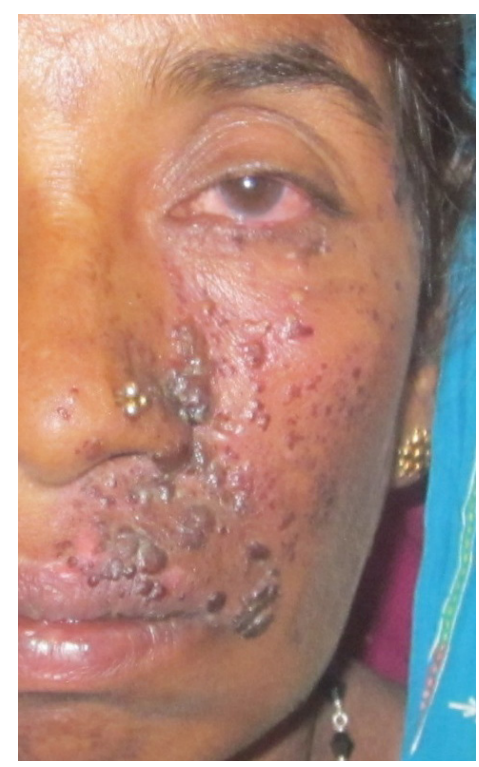

Fig.2: Maxillary involvement with Hutchison sign and limbal phlycten.

can still occur and is likely to be present if the patient reports reduced vision, eye pain or photophobia. Additionally, reduced corneal sensation is a useful sign of ocular involvement. Thus, urgent consultation with an ophthalmologist is indispensable in all cases of herpes zoster with Hutchinson sign. Early treatment can prevent ophthalmologic complications and post-herpetic neuralgia [6]. 
In the present cases, the corneal involvement can be attributed to transverion of zoster virus by direct neural connection and/or by direct spread through the tissues to other nerves. There are established rami communications between nasal branches of maxillary nerve with external nasal branches of the anterior ethmoidal nerve (branch of nasociliary nerve). These neuronal connections may explain the involvement of cornea with isolated maxillary zoster. Occasionally lacrimal nerve is absent and is replaced by zygomaticotemporal branch of maxillary nerve, sometimes conversely [7].

\section{Conclusion}

In our cases there could be a possibility of malinnervation of cornea with a branch of the maxillary nerve. Maxillary zoster may sometimes precede ophthalmic zoster and zoster ophthalmicus may occur without skin rashes, involving only the cornea or iris. All cases of zoster infestation should be referred to an ophthalmologist to prevent sequelae like corneal opacity.
Contributors: SD: manuscript writing and the conception of the work; GM was responsible for revising it critically. SD will act as a study guarantor. All authors approved the final version of this manuscript and are responsible for all aspects of this study.

Funding: None; Competing interests: None stated.

\section{References}

1. Ostler HB, Thygeson P. The ocular manifestations of herpes zoster, varicella, infectious mononucleosis and cytomegalovirus disease. Surv Ophthalmol. 1976;21:148.

2. Patil S, Srinivas K, Reddy SBH, Gupta M. Prodromal herpes zoster mimicking odontalgia- A diagnostic challenge. Ethiop J Health Sci. 2013;23:73-77.

3. Tandon MP, Verma SK. Herpes zoster maxillaries: A case report. Indian J Ophthalmol. 1987;35:160-161.

4. Hutchinson J. A clinical report on herpes zoster frontalis ophthalmicus (shingles affecting the forehead and nose). R Lond Ophthalmic Hosp Rep. 1865;5:191215.

5. Zaal MJ, Völker-Dieben HJ, D'Amaro J. Prognostic value of Hutchinson's sign in acute herpes zoster ophthalmicus. Graefes Arch Clin Exp Ophthalmol. 2003;241:187-191.

6. Matsuura H, Senoo A. Herpes zoster and Hutchinson's sign. QJM: An International Journal of Medicine. 2018;111:483.

7. Jain S, Rathore MK. Maxillary zoster with corneal involvement. Indian J Ophthalmol. 2004;52:323. 